\title{
BMJ Open Guideline deviation and its association with specific chronic diseases among patients with multimorbidity: a cross- sectional cohort study in a care management setting
}

Chandra J Cohen-Stavi (D) , ${ }^{1,2}$ Shmuel Giveon, ${ }^{3}$ Calanit Key, ${ }^{4}$ Tchiya Molcho, ${ }^{4}$ Ran Balicer, ${ }^{1,5}$ Efrat Shadmi ${ }^{1,2}$

To cite: Cohen-Stavi CJ, Giveon S, Key C, et al. Guideline deviation and its association with specific chronic diseases among patients with multimorbidity: a crosssectional cohort study in a care management setting. BMJ Open 2021;11:e040961. doi:10.1136/ bmjopen-2020-040961

- Prepublication history and supplemental material for this paper is available online. To view these files, please visit the journal online (http://dx.doi. org/10.1136/bmjopen-2020040961).

Received 06 July 2020 Revised 19 November 2020 Accepted 09 December 2020

Check for updates

(C) Author(s) (or their employer(s)) 2021. Re-use permitted under CC BY-NC. No commercial re-use. See rights and permissions. Published by BMJ.

For numbered affiliations see end of article.

Correspondence to Dr Chandra J Cohen-Stavi; chandrac0278@gmail.com

\section{ABSTRACT}

Objectives To assess whether the extent of deviation from chronic disease guideline recommendations is more prominent for specific diseases compared with combinedcare across multiple conditions among multimorbid patients, and to examine reasons for this deviation.

Design A cross-sectional cohort.

Setting Multimorbidity care management programme across 11 primary care clinics.

Patients Patients aged 45-95 years with at least two common chronic conditions, sampled according to being new ( $\leq 6$ months) or veteran ( $\geq 1$ year) to the programme. Main outcome measures Deviation from guidelinerecommended care was measured for each patient's relevant conditions, aggregated and stratified across disease groups, calculated as measures of 'diseasespecific' guideline deviation and 'combined-care' (all conditions) guideline deviation for: atrial fibrillation, congestive heart failure, chronic kidney disease, chronic obstructive pulmonary disorder, depression, diabetes, dyslipidaemia, hypertension and ischaemic heart disease. Combined-care deviation was evaluated for its association with specific diseases. Frequencies of previously derived reason types for deviation (biomedical, patient personal and contextual) were reported by nurse care managers, assessed across diseases and evaluated for their association with specific diseases.

Results Among 204 patients, disease-specific deviation varied more (from $14.7 \%$ to $48.2 \%$ ) across diseases than combined-care deviation (from $14.7 \%$ to $25.6 \%$ ). Depression and diabetes were significantly associated with more deviation (mean: $6 \%$ (95\% Cl: $2 \%$ to $10 \%$ ) and 5\% (95\% Cl: $2 \%$ to $9 \%$ ), respectively). For some conditions, assessments were among small patient samples. Guideline deviation was often attributed to non-disease-specific reasons, such as physical limitations or care burden, as much as disease-specific reasons, which was reflected in the likelihood for guideline deviation to be due to different types of reasons for some diseases.

Conclusions When multimorbid patients are considered in disease groups rather than as 'whole persons', as in many quality of care studies, the cross-cutting factors in their care delivery can be missed. The types of reasons
Strengths and limitations of this study

- This study measures guideline deviation for multiple sets of single chronic disease guideline recommendations and investigates the reasons for this deviation among multimorbid patients.

- The study contrasts descriptive assessments, typical in quality of care studies, with multivariable evaluations on a patient level, adjusting for various co-occurring conditions and other patient characteristics.

- While a substantial number of chronic conditions and corresponding guideline-recommended care processes were examined, the number of patients in many of the disease groups was small, given the overall sample size of 204 patients.

- Reasons for guideline deviation reported by nurse care managers were mostly based on second-hand accounts of the decisions made by either the physician or the patient.

more likely to occur for specific diseases may inform improvement strategies.

Trial registration number NCT01811173; Pre-results.

\section{INTRODUCTION}

Evidence-based clinical guidelines and their use in quality of care assessments suggests that there is a certainty to caring for illness-a 'right way' to do things-when in reality, there is a lot of variability and uncertainty involved in care delivery. ${ }^{1}$ This variability is particularly pronounced for patients with multiple chronic conditions (also referred to as multimorbidity) in primary care settings, for whom the multiplicity of conditions is not merely an additive sum of each individual condition. ${ }^{2}$ Therefore, while disease-focused guidelines for chronic illness provide a benchmark to guide practice and improve care quality, 
there is growing acknowledgement that high quality of care for managing multimorbidity may not simply entail performing all guideline recommendations for each specific disease. ${ }^{3}$

Recommendations based on medical evidence do not necessarily align with individual patients' unique clinical and personal circumstances and preferences. ${ }^{4-6}$ General practitioners have described this misalignment as the map not matching the terrain, with guidelines being 'reductionist' representations rather than encompassing 'complexity-based medicine'. ${ }^{7}$ Care decisions relevant to these patients, such as which treatments should not be given due to interactions, which interventions lack clear clinical benefit in light of co-occurring care demands, when should treatment be discontinued or at what point is care too burdensome, are often not incorporated into disease guidelines. ${ }^{4-6}$ While several non-disease-specific practice guidelines have been developed in recent years, ${ }^{8-12}$ this guidance has yet to make it into the infrastructure supporting quality of care strategies.

Therefore, a majority of quality of care studies have assessed the extent of guideline or quality indicator adherence for single diseases, preventive care or a few key chronic disease indicators, ${ }^{13}$ reflective of disease-centric professional standards, without necessarily accounting for what matters to patients. ${ }^{14-17}$ They report wide ranges of adherence from $10 \%$ to $80 \%$ dependent on the disease, target measures (ie, prescribing of medications, care processes, referrals, clinical marker achievement, etc) and context (ie, inpatient setting, primary care setting, one institution vs multiple, etc). ${ }^{819}$ While patients included in quality of care assessments may have other conditions than those for which adherence to quality indicators are being measured, disease-focused descriptive examination of guideline deviation considers disease groups as mutually exclusive rather than recognising the 'whole patient'. This fails to represent the management interrelatedness and the extent of care overlap that occurs in patients with multiple conditions. These quantified proportions of guideline deviation also only provide a partial understanding of what is happening in care delivery, without investigating the reasons behind this deviation.

Reasons for guideline deviation for patients with multimorbidity can be related to aspects of disease, such as the potential risk of serious biomedical interactions or complications of specific conditions and treatments, or to non-disease-specific, general aspects of the patient or context, such as extensive care burdens, physical or cognitive limitations, patient personal preferences, systemic financing structures or organisational policies. ${ }^{20-22}$ Many have cited that deviation from guidelines often occurs because of the overall combination of multiple conditions and not disease-specific factors. ${ }^{23}{ }^{24}$ Furthermore, care that is not concordant with guidelines may be due to decisions of the clinician not referring care (eg, not sending a patient to do a blood test or not prescribing a medication) or the patient not completing the referred care (eg, not doing the blood test or purchasing the medication). Numerous studies have addressed the challenges of applying guidelines to multimorbidity care conceptually through qualitative assessments of clinicians' general impressions and perspectives ${ }^{2526}$; however, there is a lack of practice-based evidence to demonstrate and characterise the extent to which care for multimorbid patients deviates from disease-centric quality assessment frameworks and why.

In order to address this gap, the current study aims to assess whether guideline deviation occurs more for certain diseases versus as a general attribute characterising all of the conditions and circumstances of multimorbid patients ('combined-care'). This is done by examining the extent of disease-based guideline deviation for multimorbidity patients and reasons for this deviation across nine common chronic conditions. It builds on prior work that evaluated the extent to which care was completed according to guidelines for multimorbid patients in a care management programme, and that derived a taxonomy of types of reasons why care deviates from guidelines. ${ }^{27} 28$

By first comparing disease-specific care (disease-related recommended care for each patient aggregated for all patients with that disease) and combined care (all care related to the relevant of nine conditions for each patient) across patient disease groups, this study aims to answer the question of whether multimorbid patients with any of nine specific diseases are on average more likely not to complete guideline-recommended care versus other guideline-recommended care for their other conditions. It then further evaluates whether specific conditions or other patient characteristics are associated with combinedcare deviation from guideline recommendations. Finally, the reasons why care deviates from guidelines elucidate whether disease-specific or non-disease-specific aspects are driving multimorbidity care decisions, which is important in helping to inform effective care quality improvements. Therefore, this study additionally examines the frequency of reasons behind guideline deviation across common chronic conditions and the association between the likelihood of types of reasons (related to biomedical, patient, healthcare context or other reasons) and each disease.

\section{METHODS}

\section{Setting}

This study was conducted as part of a primary care nursephysician team-based care management intervention at Clalit Health Services in Israel. ${ }^{27}$ The intervention, called the Comprehensive Care for Multimorbid Adults Project (CC-MAP), is a multi-site controlled trial, across 12 primary care clinics in Clalit. ${ }^{29}$ The specially trained nurses of the programme play an important role as care managers, helping to develop a care plan with the primary care physicians and providing regular follow-up consultations with the patients (on average every 1-3 months). Based on these consultations and ongoing coordination with multiple physicians, they are aware of the clinical, 
contextual and patient-related aspects of each patient's chronic care. The care management setting affords the opportunity to evaluate what is done in clinical practice in collaboration with the treating nurses of the programme, which would not be feasible in usual primary care practice due to resource and time constraints of primary care clinicians.

Adults enrolled in the CC-MAP are aged 45-95 years, have three or more chronic conditions and a morbidity index risk (represented by the Adjusted Clinical Groups morbidity index $)^{30}$ above a previously determined threshold of $6 \%$ risk for future total costs. ${ }^{31}$ Nine of the most prevalent chronic conditions in Clalit's population were determined to be the focus conditions of this study (atrial fibrillation ( $\mathrm{AF}$ ), congestive heart failure (CHF), chronic kidney disease (CKD), chronic obstructive pulmonary disorder (COPD), depression, diabetes, dyslipidaemia, hypertension and ischaemic heart disease (IHD)), based on the fact that abbreviated guideline recommendation brief references were developed as part of the CC-MAP for these nine conditions. ${ }^{27}$ Sets of key care processes were established in the prior study based on these abbreviated quick guideline references that are an integration of care recommendations for each disease from sets of international guidelines and internal Clalit protocols, with considerations for co-existing chronic conditions (see online supplemental table S1 in CohenStavi et al 2020). A total of 44 guideline-recommended care processes for managing patients' existing diagnoses, including lifestyle, follow-up tests, medications, counselling and procedure interventions, and specialist referrals (table 1), were evaluated for the extent to which they were completed over a 1year timeframe for this multimorbid patient population. ${ }^{27}$ Patients' electronic medical records were reviewed by treating CC-MAP nurses for the relevant guideline-recommended care processes related to the nine focus conditions.

The previously derived taxonomy of reasons was used in a data collection instrument, through which 11 CC-MAP nurses were asked during multiple data collection meetings to provide reasons why each care process was either not referred by the clinician or not completed by the patient if it was referred by the clinician (CohenStavi et al, 2020). Mixed methods evaluation of reasons why care deviates from clinical guidelines among patients with multimorbidity. They provided these reasons based on their knowledge as the care managers of each of their patients in the study sample, given that they are in close contact with the primary care physicians about patients' clinical care needs and have regular consultations with the patients. Nurses reviewed each patient's electronic health file and their notes within the files to remind themselves of each patient's circumstances. Sometimes more than one reason was provided by the nurses for why a care process was not completed, reflecting the complexity and multi-dimensional nature of care delivery and patient adherence. It was previously found that patient personal reasons and contextual reasons (such as the patient has no time, is not interested or has a large care burden, or care is not available) were common, as well as, biomedical reasons for explaining why multimorbidity care deviates from guidelines.

\section{Study design}

The current study was a cross-sectional cohort study of patients sampled from the ongoing CC-MAP intervention, which is a design involving cross-sectional data collection and retrospective.$^{32}$ Nurses retrospectively examined the electronic health records of patients to determine if care was received and referred according to guideline recommendations for the nine focus conditions over a 1-year period prior to the index date, which was different for each patient based on the date of data collection (anytime from June 2017 to December 2017). Baseline was defined as 1 year prior to each patient's index date. Some care processes are recommended by guidelines to be completed several times a year, and, with the exception of medication prescribing and purchases, care completion was assessed based on whether the care was referred or completed at least partially any time within the last year. $^{27}$

\section{Patient study population}

Patients in the CC-MAP were adults aged 45-95 years as described above. The current study sampled patients from the 964 patients enrolled in the second phase of the CC-MAP as of September 2017, who had at least two chronic conditions of the focus conditions (with dyslipidaemia not used for the inclusion criteria because it is sometimes considered a precondition). There were 295 patients who were in the CC-MAP at least 1 year (considered 'veteran' to the programme) or less than 6 months (considered 'new' to the programme), from which patients were randomly sampled by clinic. Inclusion was stopped at 215 patients, to allow for a balanced number of veteran versus new patients. The final sample included 204 patients, with 11 patients excluded because their information was used in the pilot phases of the study. Further details on the patient population have been described in the prior study. ${ }^{27}$ This research was reviewed and approved by the Clalit Research Ethics Committee and the institutional review board at the University of Haifa. Patient consent was received as part of the CC-MAP intervention trial.

\section{Patient and public involvement}

Patients were not involved in the design, management or reporting of this study.

\section{Definitions}

Assessments of the extent of guideline deviation and reasons for deviation were based on comparing diseasespecific care for patients who have each of the nine chronic conditions to what we refer to as 'combined care', which is the amalgamation of guideline-recommended 


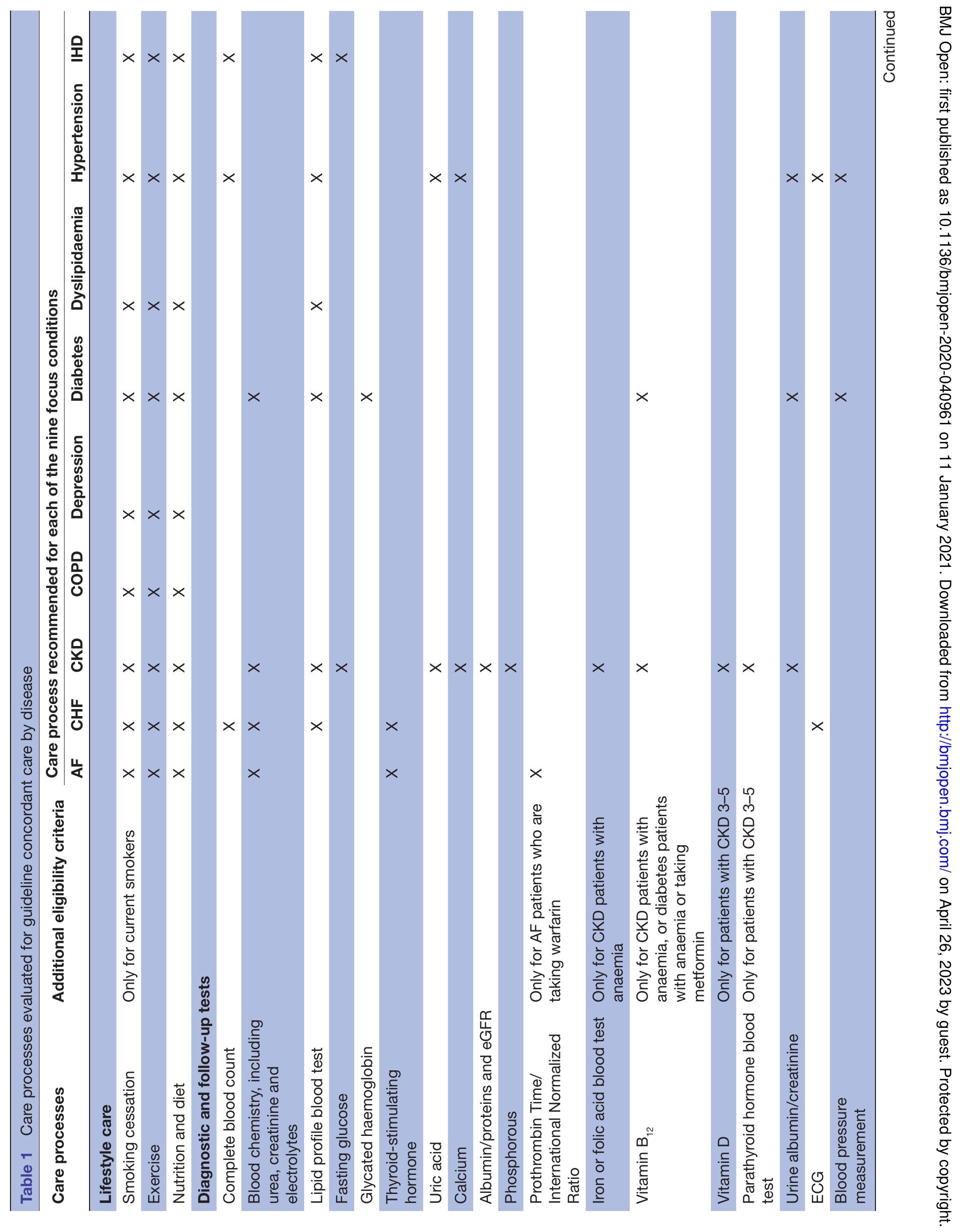




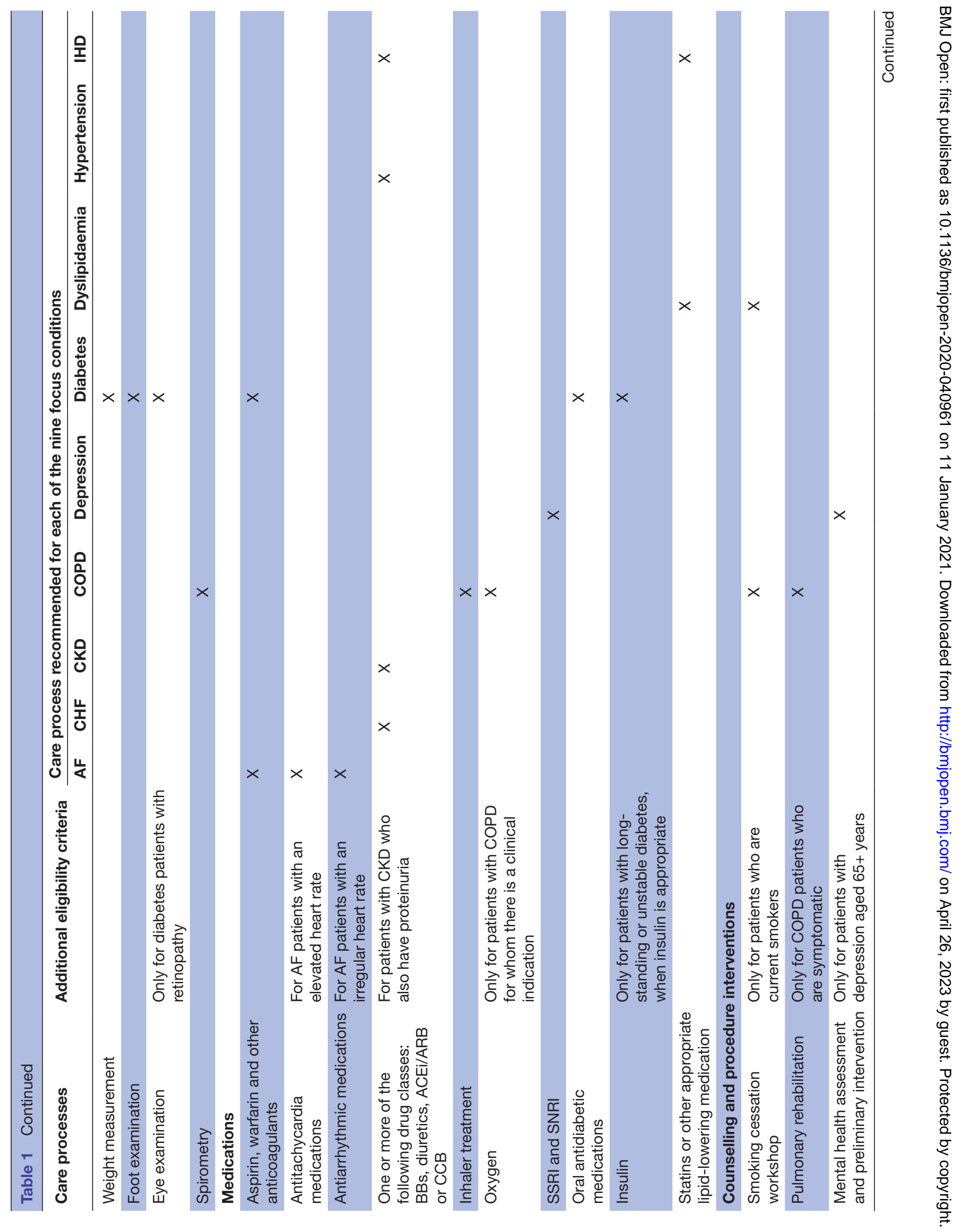




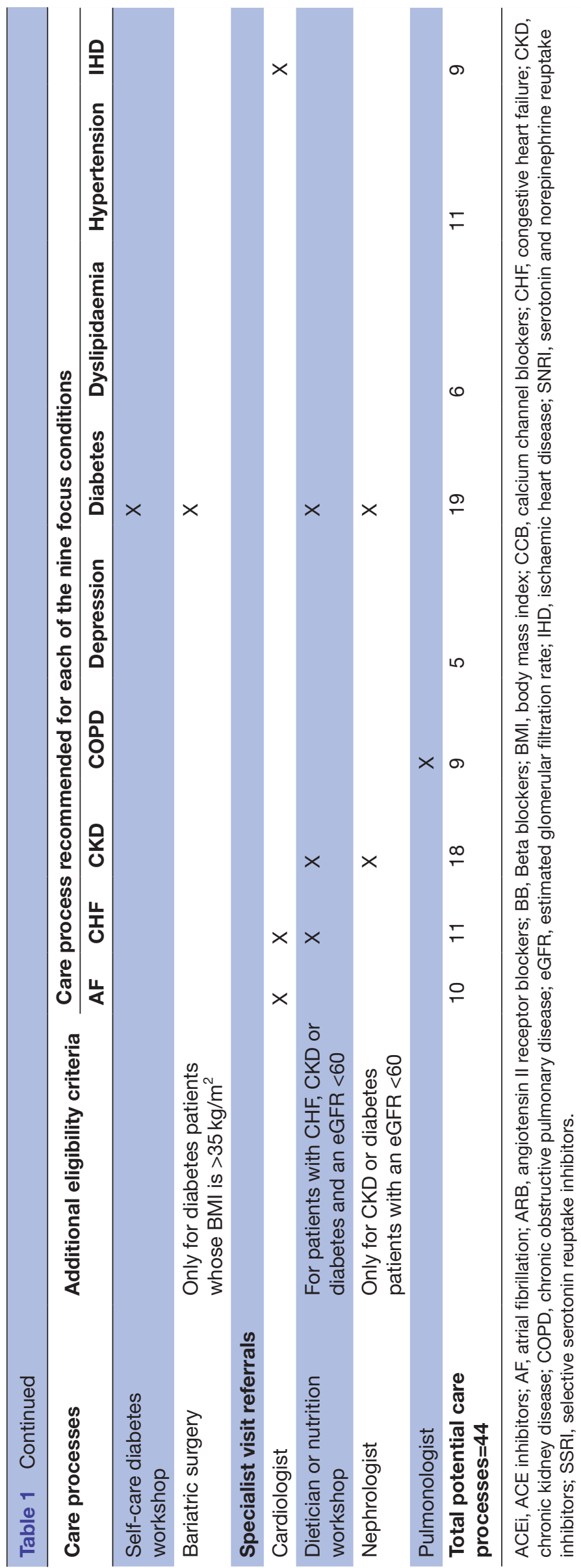

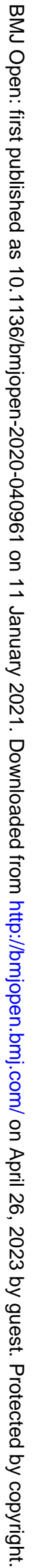


care for all relevant conditions of the nine focus conditions. For example, if a patient has diabetes, depression, hypertension and IHD, that patient was counted in each of the four disease groups, examining his or her diabetesspecific guideline-recommended care ('disease-specific care') compared with the guideline-recommended care for all four of the chronic conditions ('combined care'), and so forth for all four conditions. Therefore, patients are included in as many disease groups for which they have conditions (of the nine focus conditions). Combined-care defined as such is intended to represent the multiplicity and overlap of guideline-recommended care processes (without duplication) that make up a multimorbid patient's care management.

The guideline deviation measures were calculated on a per-patient basis and subsequently aggregated to the entire disease patient group. Combined guideline deviation was calculated as the proportion of guideline-recommended care that was not completed or received (owing to clinicians not referring it or patients not completing referred care) out of all relevant care processes recommended for each patient based on his or her set of chronic conditions (of the nine conditions). For example, if a patient completes 6 out of 10 recommendations for diseases X, Y and $\mathrm{Z}$, then that patient has $40 \%$ combined-care guideline deviation. Disease-specific guideline deviation was calculated as the proportion of guideline-recommended care that was not completed or received out of the total relevant care processes recommended for the specific disease. Clinical eligibility criteria were considered as part of this assessment in addition to chronic diagnoses, to determine the relevance of care processes for each patient based on nurses' review of the patients' files. For example, if a specialist referral is recommended only for patients who have diabetes and estimated glomerular filtration rate $($ eGFR) $<60$, then it would only be counted as one of the relevant care processes for all of the diabetes patients with an eGFR $<60$, and not for those whose eGFR $>60$.

The previously developed taxonomy of biomedical, patient personal and context reason categories encompassed the following specific reasons: biomedical reasons such as disease-drug interactions, no clinical need, side effects, physical limitations and cognitive limitations; patient personal reasons like the patient is not interested, the patient has a large care burden and the patient lacks the support; and contextrelated reasons such as missed care, efficiency drivers of the organisation, and service or care not available. Contextual reasons can be related to the organisational or health system drivers that influence care decisions, including clinical and cost-saving policies of the healthcare organisation and financing structures of the health system that determine patients' co-pays based on disability or welfare status. The full set of reasons are listed in online supplemental table S1 in the supplement. Types of reasons were assessed for disease-specific care as well as for combined-care across patient disease groups. A dichotomised variable for each type of reason category (biomedical, patient personal and context) was created per patient, for example, $0=$ no biomedical reasons indicated or $1=$ any biomedical reason indicated.

Age was categorised into three groups: $<65$ years, $65-75$ years and 76 years and older. The number of chronic conditions identified for each patient in the study comprised a potential set of 16 chronic conditions based on a list of major chronic conditions outlined by the Centers for Medicare and Medicaid Services. ${ }^{33}$ Number of chronic conditions were grouped as $2-4$ of the 16 conditions, $5-6$ conditions and 7-10 conditions.

\section{Analysis}

Deviation from guidelines

Average proportions of disease-specific care deviation compared with combined-care deviation were reported out of all relevant disease-specific and combined guideline-recommended care for patients with each of the nine chronic conditions. Because lifestyle care processes are common to all of the nine focus conditions' sets of guideline-recommended care processes, a sensitivity analysis was performed assessing guideline deviation proportions excluding the lifestyle-related care processes (smoking cessation, diet and exercise). Linear regression models were used to evaluate if patients' specific chronic diseases and were associated with combined-care guideline deviation, adjusted for age, sex and number of chronic conditions.

\section{Reasons for guideline deviation}

Frequencies of reasons explaining why care deviated from guidelines were reported for disease-specific care and combined care among the nine patient disease groups. To test whether specific diseases are significantly associated with the likelihood for a certain type of reason being attributed to guideline deviation, unadjusted and adjusted logistic regression models were run for each of the four dichotomised reason type dependent variables for each of the nine chronic conditions. The models were adjusted for the number of chronic conditions, which was the variable used to represent general multimorbidity. Analyses that were conducted to test several of the models adjusted for age and sex yielded largely similar results. Therefore, we decided to focus on adjustment for multimorbidity (represented as number of chronic conditions) only. R V.3.6.2 was used for analyses. ${ }^{34}$

\section{RESULTS}

Among the 204 patients with multimorbidity included in the study, $50(24.5 \%)$ patients had AF, $29(14.2 \%)$ had CHF, 49 (24.0\%) had CKD, $31(15.2 \%)$ had COPD, 38 (18.6\%) had depression, $128(62.7 \%)$ had diabetes, 178 (87.3\%) had dyslipidaemia, 181 (88.7\%) had hypertension and $90(44.1 \%)$ had IHD (table 2). Mean age among different patient disease groups ranged from 70.6 years (SD: 11.1 years) in patients with depression, to 77.7 years (SD: 7.1 years) among patients with AF. Patients with CHF 


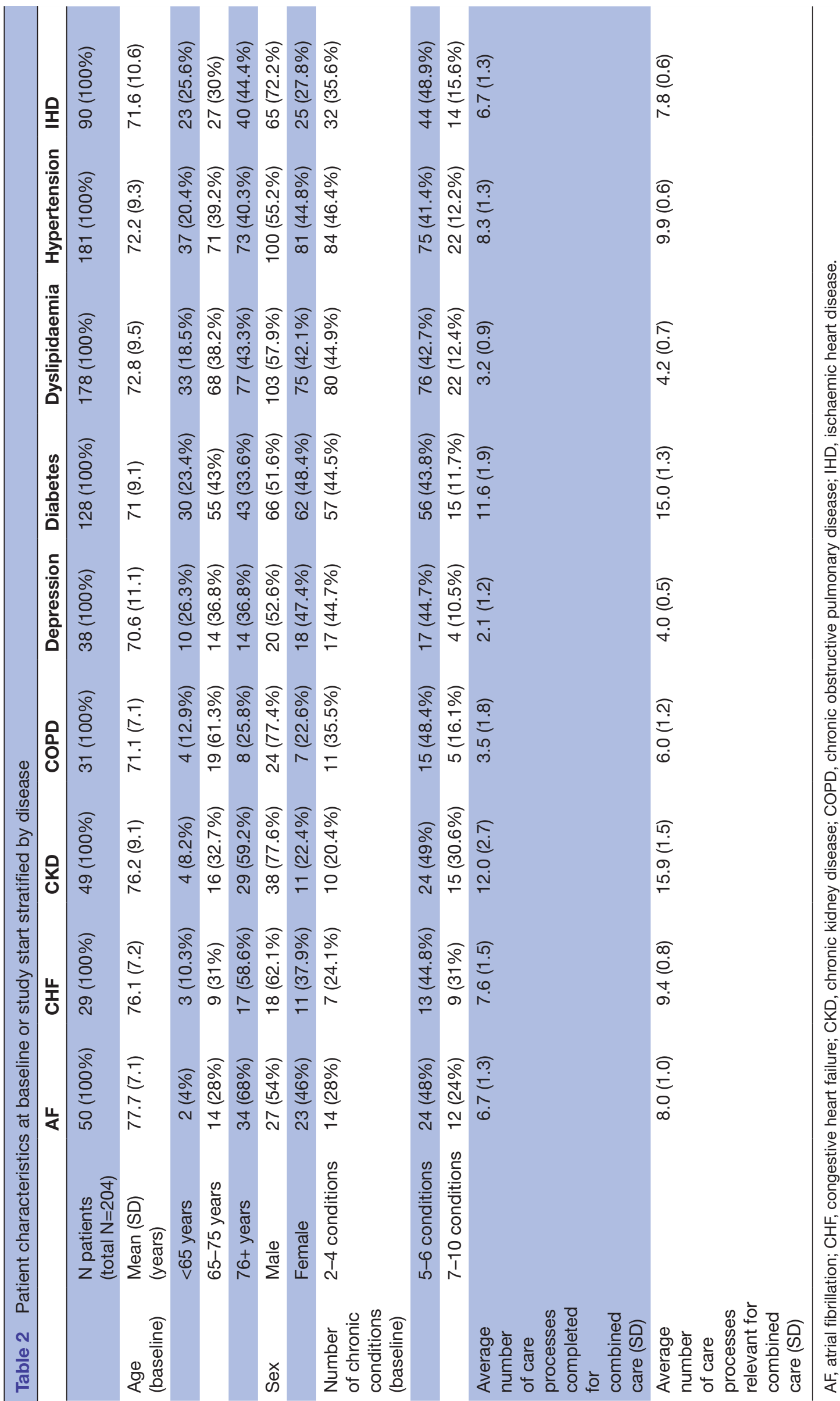


Care that deviates from guideline recommendations: mean diseasespecific care vs. mean combined care for patients with that disease

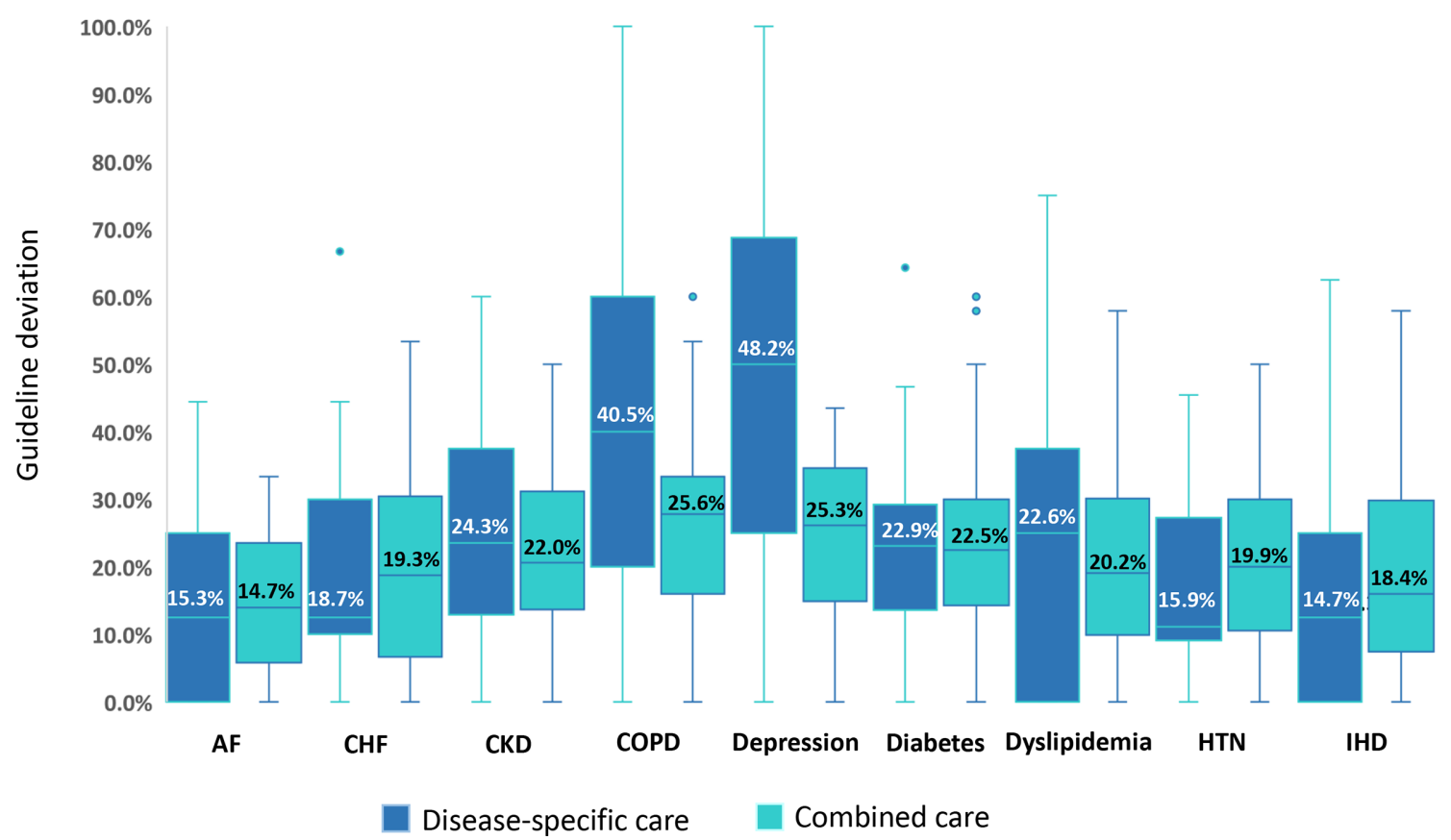

Figure 1 Care that deviates from guideline recommendations: mean disease-specific care vs mean combined care for patients with that disease. AF, atrial fibrillation; CHF, congestive heart failure; CKD, chronic kidney disease; COPD, chronic obstructive pulmonary disease; HTN, hypertension; IHD, ischaemic heart disease.

and CKD had the largest proportions of the most chronic conditions (7-10 conditions), with $31 \%$ and $30.6 \%$, respectively. The average number of care processes completed for combined care (numerator) ranged from 2.1 (SD: 1.2) for depression to 12.0 (SD: 2.7) for CKD; and the average number of care processes relevant for combined care (denominator) ranged from 4.0 (SD: 0.5) for depression to 15.9 (SD: 1.5) for CKD.

\section{Deviation from guidelines}

In the study population, the average deviation from guidelines was $20.8 \%$ (SD 12\%) (results from previously published study). ${ }^{27}$ Across a majority (5/9) of the conditions, the proportion of provided care that deviated from guidelines was similar between disease-specific care and combined care (figure 1). Deviation from guidelines for disease-specific care ranged from $14.7 \%$ for IHD-related care to $48.2 \%$ for depression-related care; and proportions ranged from $14.7 \%$ for combined care among AF patients to $25.6 \%$ for combined care among COPD patients. COPD-specific care and depression-specific care had the highest proportions of deviation from guidelines, with $40.5 \%$ and $48.2 \%$ deviations, respectively. The proportions of guideline deviation for combined care in these same two patient disease groups were $25.6 \%$ and $25.2 \%$, respectively. The lowest proportions of guideline deviation were found for disease-specific care for AF, hypertension and IHD with $15.2 \%, 15.9 \%$ and $14.7 \%$, respectively. For hypertension and IHD, the disease-specific guideline deviation was lower than for combined care with $19.9 \%$ and $18.4 \%$, respectively. In the sensitivity analysis, which excluded lifestyle care processes as part of the common denominator across all guideline-recommended disease care, some of the discrepancies between disease-specific and combined care were more pronounced than in the measures that included lifestyle care, such as for CHF, hypertension and IHD. Meanwhile, for other diseases, the discrepancy was reversed without lifestyle processes, with combined-care deviation being greater than diseasespecific care deviation, such as observed among patients with dyslipidaemia (online supplemental figure S1).

In the adjusted model, significant associations were found between having diagnoses of $\mathrm{AF}$ and hypertension and lower likelihood for combined-care guideline deviation, with a mean difference of $8 \%$ lower likelihood to have guideline deviation for patients with $\mathrm{AF}$ or hypertension diagnoses than with other diagnoses. Whereas, significant associations were found between having diagnoses of depression and diabetes and increased likelihood of combined-care guideline deviation (table 3). No significant association was found between increased guideline deviation and other diagnoses or patient characteristics.

\section{Reasons for guideline deviation}

For combined-care deviation, between $30 \%$ and $40 \%$ of the time biomedical reasons were attributed to guideline deviation, among $60 \%-90 \%$ of patients across disease groups (figure 2A, B). There was greater variation in the prevalence of patient-related and context-related reasons, ranging from $19 \%$ to $35 \%$ for patient-related reasons 
Table 3 Unadjusted and adjusted linear regression for the association between combined-care guideline deviation and specific diseases and patient characteristics among multimorbid patients

\begin{tabular}{|c|c|c|c|c|}
\hline Covariates & Unadjusted & $95 \% \mathrm{Cl}$ & $\begin{array}{l}\text { Adjusted linear regression } \\
\text { estimate }\end{array}$ & $95 \% \mathrm{Cl}$ \\
\hline \multicolumn{5}{|l|}{ Diseases } \\
\hline $\mathrm{AF}$ & -0.08 & -0.12 to -0.04 & -0.07 & -0.11 to -0.02 \\
\hline $\mathrm{CHF}$ & -0.02 & -0.07 to 0.03 & 0.01 & -0.04 to 0.06 \\
\hline CKD & 0.02 & -0.02 to 0.06 & 0.04 & -0.01 to 0.08 \\
\hline COPD & 0.06 & 0.01 to 0.10 & 0.03 & -0.02 to 0.08 \\
\hline Depression & 0.05 & 0.01 to 0.10 & 0.06 & 0.02 to 0.10 \\
\hline Diabetes mellitus & 0.05 & 0.01 to 0.08 & 0.05 & 0.02 to 0.09 \\
\hline Dyslipidaemia & -0.05 & -0.10 to 0.00 & -0.04 & -0.08 to 0.01 \\
\hline Hypertension & -0.08 & -0.13 to -0.02 & -0.08 & -0.13 to -0.03 \\
\hline IHD & -0.04 & -0.08 to -0.01 & -0.03 & -0.07 to 0.01 \\
\hline \multicolumn{5}{|l|}{ Age (years) } \\
\hline$<65$ & Reference & & Reference & \\
\hline $65-75$ & -0.04 & -0.09 to 0.01 & -0.04 & -0.08 to 0.01 \\
\hline $76+$ & -0.05 & -0.10 to -0.00 & -0.02 & -0.07 to 0.02 \\
\hline \multicolumn{5}{|l|}{ Sex } \\
\hline Female & Reference & & Reference & \\
\hline Male & 0.02 & -0.01 to 0.06 & 0.02 & -0.01 to 0.06 \\
\hline \multicolumn{5}{|l|}{ Chronic condition count } \\
\hline 2-4 chronic conditions & Reference & & Reference & \\
\hline 5-6 chronic conditions & -0.01 & -0.04 to 0.03 & 0.00 & -0.04 to 0.04 \\
\hline 7-10 chronic conditions & 0.00 & -0.06 to 0.06 & 0.02 & -0.05 to 0.08 \\
\hline Adjusted $\mathrm{R}^{2}$ & & & $0.19, p<0.001$ & \\
\hline
\end{tabular}

$\mathrm{AF}$, atrial fibrillation; $\mathrm{CHF}$, congestive heart failure; CKD, chronic kidney disease; COPD, chronic obstructive pulmonary disease; IHD, ischaemic heart disease.

among $40 \%-74 \%$ of patients and $11 \%-26 \%$ for contextrelated reasons among $47 \%-67 \%$ of patients across disease groups. For most disease groups, biomedical reasons were most prevalent, except for depression and IHD, where patient-related reasons were most prominent (figure 2A). Most of the three reason types (biomedical, patient and context) were prevalent among at least $50 \%$ of patients across disease groups with exceptions, including patient-related reasons among only $40.0 \%$ of AF patients and $48.3 \%$ of CHF patients, and context-related reasons among $47.4 \%$ of depression patients (figure 2B).

When examining the odds of various types of reasons attributed to guideline deviation adjusted for number of chronic conditions (ie, general multimorbidity), patients with COPD and diabetes had about four times significantly greater odds of having at least one biomedical reason for not performing guideline recommendations (OR: 3.79, 95\% CI: 1.09 to 13.15 and OR: $4.18,95 \%$ CI: 2.16 to 8.06 , respectively), and those with COPD or diabetes had about twofold greater odds of having a biomedical reason (OR: 2.14, 95\% CI: 1.07 to 4.27 and OR: $2.07,95 \%$ CI: 1.01 to 4.24 , respectively) (table 4). Patients with a diagnosis of IHD had significantly lower odds of having a biomedical reason (OR: $0.22,95 \%$ CI: 0.11 to 0.45$)$. Having a depression diagnosis was the only disease that was significantly associated with more than two times greater odds (OR: 2.55, 95\% CI: 1.16 to 5.62) of having patient-related reasons to explain guideline deviation. Patients with an AF diagnosis were significantly less likely to have patientrelated reasons for guideline deviation (OR: $0.33,95 \%$ CI: 0.16 to 0.68 ). Patients with diabetes were more than three times more likely to have at least one context-related reason attributed to guideline deviation.

\section{DISCUSSION}

The current study captures some of the complexity involved in multimorbidity care decisions and delivery. Among this multimorbid patient group, proportions of deviation from guidelines vary from $15 \%$ to almost $50 \%$ across diseases for disease-specific guidelinerecommended care. Disease-specific guideline deviation was considerably greater than combined-care deviation among patients with COPD and depression. When adjusted for other chronic conditions and key patient characteristics, combined care was significantly more 

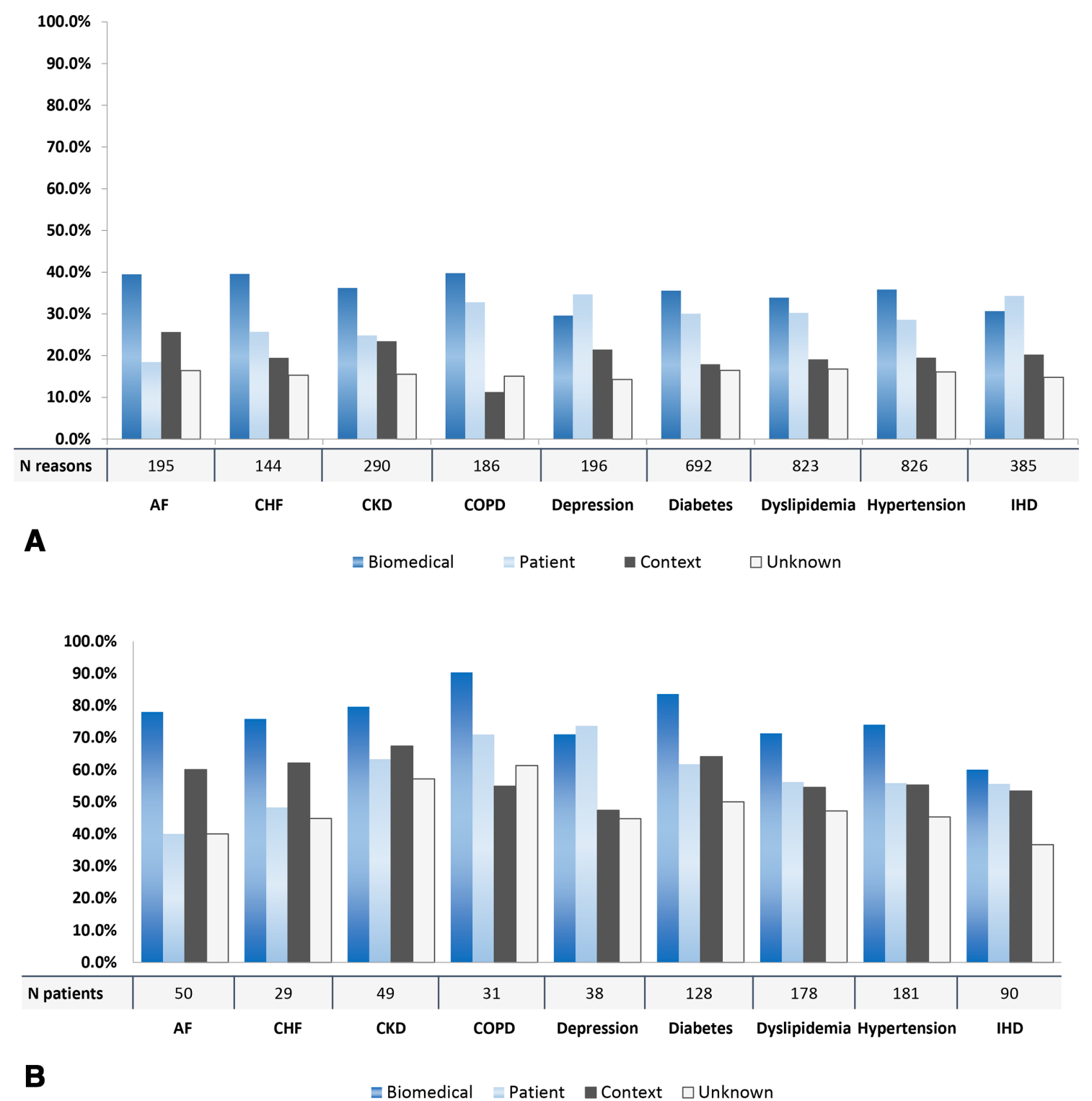

Figure 2 (A) Per cent of biomedical, patient-related and context-related reasons for deviation from combined care guidelines across patient disease groups. (B) Per cent of patients who have at least one biomedical, patient-related or context-related reason for deviation from combined care guidelines across patient disease groups. AF, atrial fibrillation; CHF, congestive heart failure; CKD, chronic kidney disease; COPD, chronic obstructive pulmonary disease; IHD, ischaemic heart disease.

likely to deviate from guidelines for multimorbid patients with some of the specific diseases, such as diabetes and depression, while there was a significant decreased likelihood of guideline deviation among patients with other diseases, such as $\mathrm{AF}$ and hypertension. For diabetes, guideline deviation was more likely to be because of biomedical and context-related reasons, and for depression, because of patient personal reasons. Among those with $\mathrm{AF}$, the reasons for deviation were significantly less likely to be due to patient personal reasons than for those who do not have AF. The reasons behind guideline deviation were often related to non-disease-specific factors, such as physical limitations, patients' lack of interest, large care burdens or contextual organisational policies just as much as disease-specific reasons such as no clinical need (for a specific care process) or biomedical risks outweighing the benefits.

When examining disease-specific guideline deviation compared with combined-care guideline deviation in the same groups of patients across different diseases, discrepancies were identified, indicating whether this deviation was due more to aspects specific to a disease or more generally to the group of patients who have that disease among other diseases. Some of the observed differences in disease-specific care versus combined care can be a function of the number of care processes and kinds of care recommended for specific diseases. For example, there are only a few guideline-recommended care processes for depression and COPD (particularly if lifestyle processes are not counted); therefore, each 
Table 4 Unadjusted and adjusted logistic regression evaluating whether specific diseases or general multimorbidity are associated with different types of reasons attributed to guideline deviation

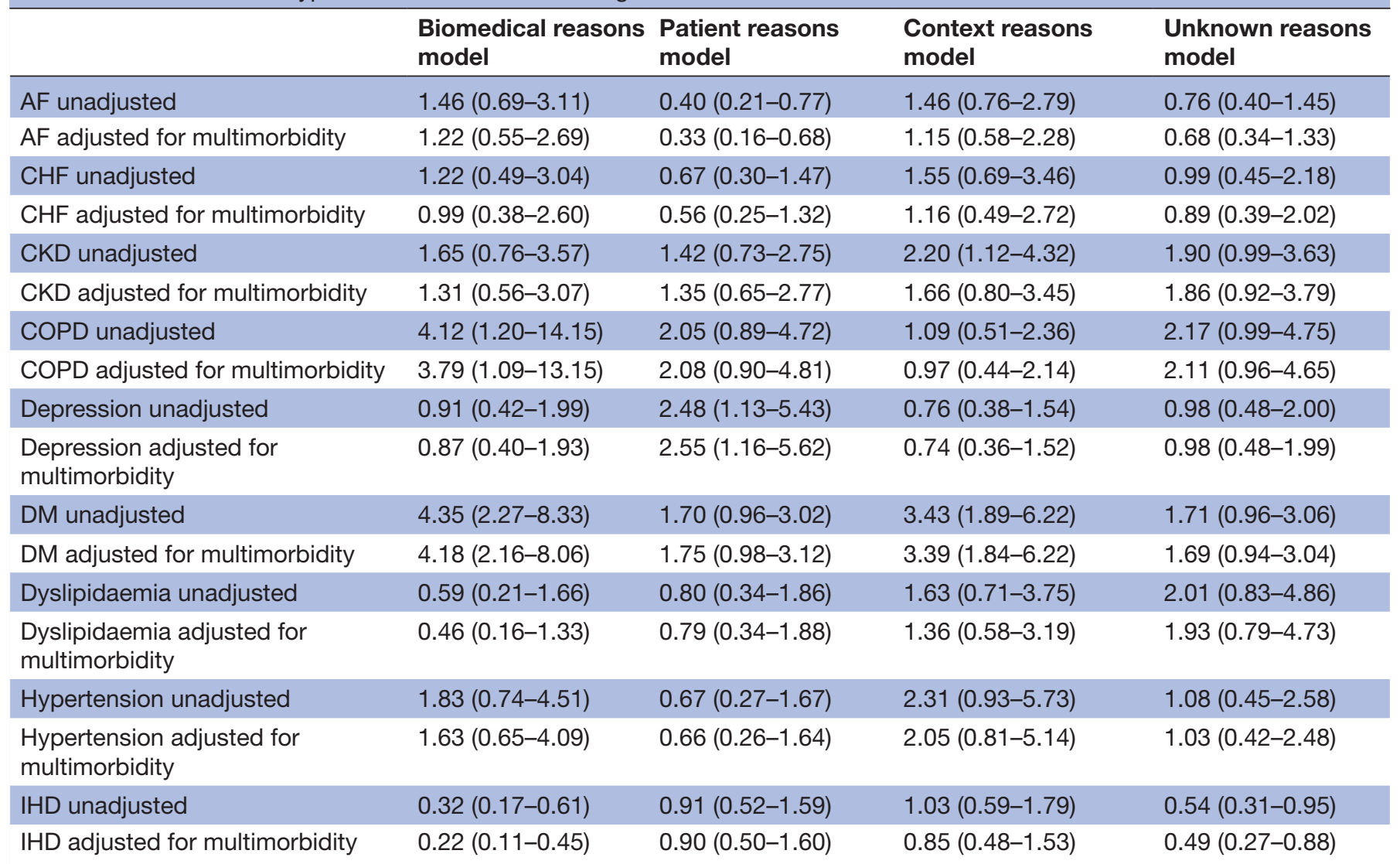

AF, atrial fibrillation; CHF, congestive heart failure; CKD, chronic kidney disease; COPD, chronic obstructive pulmonary disease; DM, diabetes mellitus; IHD, ischaemic heart disease.

deviation carries more weight in terms of its effect on the aggregated mean score. The higher proportions of disease-specific guideline deviation and greater variance between combined and diseases-specific care for COPD and depression may also be due to these diseases being discordant, in that they do not share similar pathogeneses or management strategies with other conditions. ${ }^{35}$ As such, it has been suggested that COPD is deprioritised in primary care visits, particularly among multimorbid patients, because of lack of awareness, lack of local care routines related to COPD and poor documentation. ${ }^{36}$ Patients with diseases that tend to have care management overlap, such as IHD, dyslipidaemia and hypertension, were likely to have less guideline deviation (even if the associations were borderline significant). This corroborates what has been concluded from previous literature, that quality of care appears to be higher for those with concordant conditions and lower among those with discordant conditions. ${ }^{13}$

Yet, descriptive examination of guideline deviation and reasons for deviation fail to represent the interrelatedness of concordant and discordant diseases, as well as the cross-cutting reasons for deviation that are not a function of any one specific disease. This study contrasts the descriptive assessments with multivariable evaluation on a patient level, adjusting for key clinical and demographic characteristics, and various chronic diseases, which differs from typical quality of care assessments that do not account for the other co-occurring morbidities that a patient has.

The significant association found between greater combined-care guideline deviation and diabetes or depression diagnoses may be explained by the heterogeneity of patients with diabetes or depression. ${ }^{37} 38$ Particularly, because this heterogeneity can lead to varying treatment and care management strategies that are not explicitly distinguished within disease guideline recommendations. For diabetes patients, there is an extensive and diverse set of care processes (eg, lifestyle, follow-up tests, medications, counselling and procedure interventions and specialist visits types of care) that are recommended by guidelines; yet, these are not all needed for all diabetes patients, given the varying severity and progression of disease in different patients. We found support of this based on our analyses of the reasons why care deviated from guidelines, in that many of the diabetesrelated care processes were not completed because of 'no clinical need'. Still, much of guideline-recommended diabetes care relies on the patient to make a significant effort to complete, such as different types of lifestyle and 
self-management care, for which adherence to diabetes guidelines has been shown to vary widely depending on the type of care. ${ }^{39}$ Self-management activities are also particularly challenging for multimorbid patients to complete due to physical and emotional symptoms, coping with pain and depression, difficulties in access to care and other contextual factors. ${ }^{2340}$

Our findings are also consistent with what several studies have concluded that patients with depression and co-occurring chronic conditions are less likely to have optimal treatment and more likely not to perform self-care due to varying factors, such as high risk of drug side effects, variable mood, lack of motivation and other personal challenges. ${ }^{41-43}$ Something that might be emblematic among patients with chronic morbidities and depression is that their perspectives on disease can shift over time and are often governed by emotional considerations, as was shown in a one qualitative study with patient responses such as, 'We have heard so much about the diabetes, but I have put it completely on the shelf until the mental stuff is in place, because if I cannot survive mentally, then it doesn't bloody matter how much diabetes I've got'. ${ }^{44}$

Several limitations of this study should be considered. While a substantial number of chronic conditions and corresponding guideline-recommended care processes were examined, analyses were still limited to the nine focus conditions and not all possible chronic illnesses patients had. To account for more of the multimorbidity burden, a more extensive set of chronic conditions was included in the chronic condition count covariate in adjusted analyses. Furthermore, the number of patients in many of the disease groups was small, given the overall sample size of 204 patients. Therefore, many of the diseases yielded borderline significance in adjusted analyses examining their association with guideline deviation, which might have been significant had there been more patients represented in each disease group. The study sample size was originally estimated for linear regression assumptions pertaining to the proportion of guideline deviation outcome, and, therefore, the study may have been underpowered to detect an effect in the logistic regression analyses examining the association between specific diseases and the likelihood for different types of reasons. A further limitation was that we were unable to measure the extent of overlap in care processes while employing a disease-focused assessment across patient disease groups. Future studies could build on this exploratory analysis to measure the extent of care overlap in larger multimorbid patient samples with varied disease groups.

Another limitation is that the reasons reported on by the treating nurses were (for most of the care processes) based on second-hand accounts of the decisions made by either the physician or the patient. However, the nurses' close collaboration with the primary care physicians and the extensive contact with the patients within the CC-MAP team-based care strategy, means that they have an in-depth understanding of both the clinical care decisions and patients' perspectives. Additionally, when the nurses were not familiar with the specific circumstances surrounding a care decision, they reported the reason as unknown. With this, some of the disease-specific biomedical reasons why physicians did not recommend care according to guidelines may have been underrepresented because the nurses are trained in different aspects of care often with a patient-centred focus.

Given the misalignment of disease-specific guidelines to practice care decisions for multimorbid patients, it may very well be that care that deviates from guideline recommendations constitutes high rather than poor quality of care. One review study that examined reasons for guideline or quality indicator deviation (not in multimorbid populations but for a wide spectrum of disease targets) found that the main reasons for guideline deviation were contra-indications and patient decision. ${ }^{19}$ In the current study, diagnoses of COPD, diabetes or depression were the only specific diseases that were significantly likely to be associated with biomedical, biomedical and contextual, and patient-related reasons, respectively, for guideline deviation. Whereas, many of the reasons why care was not done according to guidelines were not specific to any one disease, and were more generally related to the patient's clinical and personal circumstances, or nondisease-specific system drivers. This is consistent with many of the general challenges cited by physicians in qualitative studies examining the difficulties of applying guideline recommendations to multimorbid patients. ${ }^{25} 26$ Observations of the type of reasons more likely to occur for specific diseases may help to inform improvement strategies, such as adapting quality assessments to allow for patient preferences and circumstances among patients with depression and multimorbidity because patient-related reasons are more likely to be attributed to care deviation for these patients. Overall, this study demonstrates that there is a diversity in the types of reasons for guideline deviation that are likely to occur for a majority of patients with multimorbidity-despite the specific disease groups they may be counted in-and that broader conceptualisations of what is considered appropriate care for these patients should account for this diversity.

In summary, we have described and evaluated observations from primary care practice compared with the benchmark of disease-focused standards, and found that for some diseases, disease-related aspects are associated with either greater or less deviation from guidelines. Yet, other crosscutting aspects such as physical functioning or patients' priorities are frequently cited as factors for why guideline-recommended care was not completed. Conclusions that are drawn about the quality of care in other disease-specific studies are an artefact of the disease focus, and may, in effect, miss these crosscutting factors. The reasons why care deviate from guideline recommendations shed light on the extent of the misalignment between additive disease-focused guideline recommendations for multiple conditions and patients' actual care needs and priorities. If quality metrics would also encompass measures that reflect patients' priorities as a primary 
target for high quality, this might shift the focus of care from a disease-only orientation toward greater alignment between clinicians and patients.

\section{Author affiliations}

${ }^{1}$ Clalit Research Institute, Clalit Health Services, Tel Aviv, Israel

${ }^{2}$ The Cheryl Spencer Department of Nursing, Faculty of Social Welfare and Health Sciences, University of Haifa, Haifa, Israel

${ }^{3}$ Community Medical Division, Clalit Health Services, Tel Aviv, Israel

${ }^{4}$ Community Nursing Division, Clalit Health Services, Tel Aviv, Israel

${ }^{5}$ Public Health Department, Ben-Gurion University of the Negev, Beersheba, Israel

Acknowledgements We would like to thank all of the Comprehensive Care for Multimorbid Adults Project (CC-MAP) nurses and the larger CC-MAP clinical team and staff for their contribution to this study. Finally, we would like to thank Professor Morton Leibowitz for critically reviewing the manuscript, and Adi Berliner for advising on the statistical analyses.

Contributors All authors contributed to the conception and design of the study. CK, RB and ES obtained funding for the study. SG, CK, RB and ES provided clinical and project oversight for the study. CJC-S, TM and ES contributed to research investigation. CJC-S and ES were responsible for the data collection and curation. CJC-S and ES developed the methodology, and analysed and interpreted the data. CJC-S conducted the statistical analysis using programming software, prepared the visualisation of the data and drafted the manuscript. All authors critically reviewed the manuscript. ES is the guarantor.

Funding The study was supported by the Gertner Institute, Clalit Health Services, and in part by a grant from the Israel National Institute for Health Policy Research (grant \#2015/190). The funders did not contribute to the design, conduct of the study, the interpretation of the data or the preparation of the manuscript.

Competing interests All authors have no additional competing interests to report except for receipt of funding of this work from the organisations listed in the funding section.

Patient consent for publication Not required.

Provenance and peer review Not commissioned; externally peer reviewed.

Data availability statement № additional data are available.

Supplemental material This content has been supplied by the author(s). It has not been vetted by BMJ Publishing Group Limited (BMJ) and may not have been peer-reviewed. Any opinions or recommendations discussed are solely those of the author(s) and are not endorsed by BMJ. BMJ disclaims all liability and responsibility arising from any reliance placed on the content. Where the content includes any translated material, BMJ does not warrant the accuracy and reliability of the translations (including but not limited to local regulations, clinical guidelines, terminology, drug names and drug dosages), and is not responsible for any error and/or omissions arising from translation and adaptation or otherwise.

Open access This is an open access article distributed in accordance with the Creative Commons Attribution Non Commercial (CC BY-NC 4.0) license, which permits others to distribute, remix, adapt, build upon this work non-commercially, and license their derivative works on different terms, provided the original work is properly cited, appropriate credit is given, any changes made indicated, and the use is non-commercial. See: http://creativecommons.org/licenses/by-nc/4.0/.

\section{ORCID iD}

Chandra J Cohen-Stavi http://orcid.org/0000-0002-5190-120X

\section{REFERENCES}

1 Hargraves I, Kunneman M, Brito JP, et al. Caring with evidence based medicine. BMJ 2016;353:i3530.

2 Wei MY, Kawachi I, Okereke OI, et al. Diverse cumulative impact of chronic diseases on physical health-related quality of life: implications for a measure of multimorbidity. Am J Epidemiol 2016;184:357-65.

3 Reeve J. Scholarship-based medicine: teaching tomorrow's generalists why it's time to retire EBM. $\mathrm{Br} J$ Gen Pract 2018;68:390-1.

4 Elwyn G, Wieringa S, Greenhalgh T. Clinical encounters in the postguidelines era. BMJ 2016;353:i3200.
5 McCartney M, Treadwell J, Maskrey N, et al. Making evidence based medicine work for individual patients. BMJ 2016;353:i2452.

6 Mangin D, Heath I, Jamoulle M. Beyond diagnosis: rising to the multimorbidity challenge. BMJ 2012;344:e3526.

7 Austad B, Hetlevik I, Mjølstad BP, et al. Applying clinical guidelines in general practice: a qualitative study of potential complications. BMC Fam Pract 2016;17:92

8 American Geriatrics Society Expert Panel on the Care of Older Adults with Multimorbidity. Patient-Centered care for older adults with multiple chronic conditions: a stepwise approach from the American geriatrics Society: American geriatrics Society expert panel on the care of older adults with multimorbidity. J Am Geriatr Soc 2012;60:1957-68.

9 American Geriatrics Society Expert Panel on the Care of Older Adults with Multimorbidity. Guiding principles for the care of older adults with multimorbidity: an approach for clinicians. J Am Geriatr Soc 2012;60:E1-25.

10 Muth C, van den Akker M, Blom JW, et al. The Ariadne principles: how to handle multimorbidity in primary care consultations. BMC Med 2014:12:223.

11 National Institute for Health and Care Excellence. Multimorbidity: assessment, prioritisation and management of care for people with commonly occurring multimorbidity (NG56). NICE quideline. London, UK: National Guideline Centre, 2016.

12 O'Mahony D, O'Sullivan D, Byrne S, et al. STOPP/START criteria for potentially inappropriate prescribing in older people: version 2. Age Ageing 2015;44:213-8.

13 Valderas JM, Gangannagaripalli J, Nolte E, et al. Quality of care assessment for people with multimorbidity. J Intern Med 2019;285:289-300.

14 Mutter JB, Liaw W, Moore MA, et al. Core principles to improve primary care quality management. J Am Board Fam Med 2018;31:931-40.

15 Tinetti ME, Naik AD, Dodson JA. Moving from Disease-Centered to patient Goals-Directed care for patients with multiple chronic conditions: patient value-based care. JAMA Cardiol 2016;1:9-10.

16 Lynn J, McKethan A, Jha AK. Value-Based payments require Valuing what matters to patients. JAMA 2015;314:1445-6.

17 Santana M-J, Ahmed S, Lorenzetti D, et al. Measuring patientcentred system performance: a scoping review of patient-centred care quality indicators. BMJ Open 2019;9:e023596.

18 Willis TA, West R, Rushforth B, et al. Variations in achievement of evidence-based, high-impact quality indicators in general practice: an observational study. PLoS One 2017;12:e0177949.

19 Arts DL, Voncken AG, Medlock S, et al. Reasons for intentional guideline non-adherence: a systematic review. Int J Med Inform 2016:89:55-62

20 Bayliss EA, Bonds DE, Boyd CM, et al. Understanding the context of health for persons with multiple chronic conditions: moving from what is the matter to what matters. Ann Fam Med 2014;12:260-9.

21 Rosbach M, Andersen JS. Patient-experienced burden of treatment in patients with multimorbidity - A systematic review of qualitative data. PLoS One 2017;12:e0179916.

22 Wallace E, Salisbury C, Guthrie B, et al. Managing patients with multimorbidity in primary care. BMJ 2015;350:h176.

23 Boyd CM, Wolff JL, Giovannetti E, et al. Healthcare task difficulty among older adults with multimorbidity. Med Care 2014;52 Suppl 3:S118-25.

24 Buffel du Vaure C, Ravaud P, Baron G, et al. Potential workload in applying clinical practice guidelines for patients with chronic conditions and multimorbidity: a systematic analysis. BMJ Open 2016;6:e010119.

25 Luijks H, Lucassen P, van Weel C, et al. How GPs value guidelines applied to patients with multimorbidity: a qualitative study. BMJ Open 2015;5:e007905.

26 Sinnott C, Mc Hugh S, Browne J, et al. Gps' perspectives on the management of patients with multimorbidity: systematic review and synthesis of qualitative research. BMJ Open 2013;3:e003610.

27 Cohen-Stavi CJ, Key C, Giveon S, et al. Assessing guidelineconcordant care for patients with multimorbidity treated in a care management setting. Fam Pract 2020;37:479-85.

28 Cohen-Stavi CJ, Key C, Molcho T, et al. Mixed methods evaluation of reasons why care deviates from clinical guidelines among patients with multimorbidity. Med Care Res Rev 2020:1077558720975543.

29 Balicer RD. Comprehensive-Care for Multimorbid adults effectiveness study (CCMAP): ClinicalTrials.gov identifier: NCT01811173. 14 March 2013. Available: https://clinicaltrials.gov/ ct2/show/NCT01811173 [Accessed 5 March 2019].

30 Starfield B, Weiner J, Mumford L, et al. Ambulatory care groups: a categorization of diagnoses for research and management. Health Serv Res 1991;26:53-74. 
31 Cohen CJ, Flaks-Manov N, Low M, et al. High-Risk case identification for use in comprehensive complex care management. Popul Health Manag 2015;18:15-22.

32 Hudson JI, Pope HG, Glynn RJ. The cross-sectional cohort study: an underutilized design. Epidemiology 2005;16:355-9.

33 US Centers for Medicare and Medicaid Services. Chronic condition data Warehouse module 2. Available: http://resdac.umn.edu/sites/ resdac.umn.edu/files/Module\%202\%20-\%20Data\%20Structure\% 20and\%20Contents\%20-\%20Slides_0.pdf [Accessed 11 August 2018].

$34 \mathrm{R}$ Foundation for Statistical Computing. $R$ : A language and environment for statistical computing. [program]. 3.6.2 version. Vienna, Austria: R Foundation for Statistical Computing, 2019.

35 Zulman DM, Asch SM, Martins SB, et al. Quality of care for patients with multiple chronic conditions: the role of comorbidity interrelatedness. J Gen Intern Med 2014;29:529-37.

36 Sandelowsky H, Hylander I, Krakau I, et al. Time pressured deprioritization of COPD in primary care: a qualitative study. Scand J Prim Health Care 2016;34:55-65.

37 Fried El. Moving forward: how depression heterogeneity hinders progress in treatment and research. Expert Rev Neurother 2017;17:423-5.

38 Karalliedde J, Gnudi L. Diabetes mellitus, a complex and heterogeneous disease, and the role of insulin resistance as a determinant of diabetic kidney disease. Nephrol Dial Transplant 2016;31:206-13.

39 Oude Wesselink SF, Lingsma HF, Robben PBM, et al. Guideline adherence and health outcomes in diabetes mellitus type 2 patients: a cross-sectional study. BMC Health Serv Res 2015;15:22.

40 Liddy C, Blazkho V, Mill K. Challenges of self-management when living with multiple chronic conditions: systematic review of the qualitative literature. Can Fam Physician 2014;60:1123-33.

41 Alvarado-Martel D, Ruiz Fernández M Ángeles, Cuadrado Vigaray $\mathrm{M}$, et al. Identification of psychological factors associated with adherence to self-care behaviors amongst patients with type 1 diabetes. J Diabetes Res 2019;2019:1-9.

42 Doos L, Roberts EO, Corp N, et al. Multi-Drug therapy in chronic condition multimorbidity: a systematic review. Fam Pract 2014;31:654-63.

43 Egede LE, Ellis C, Grubaugh AL. The effect of depression on selfcare behaviors and quality of care in a national sample of adults with diabetes. Gen Hosp Psychiatry 2009;31:422-7.

44 Kristensen MAT, Guassora AD, Arreskov AB, et al. 'I've put diabetes completely on the shelf till the mental stuff is in place'. How patients with doctor-assessed impaired self-care perceive disease, self-care, and support from general practitioners. A qualitative study. Scand $J$ Prim Health Care 2018;36:342-51. 